artículos derivados de proyectos de investigación 

CUAD. CONTAB. / BOGOTÁ, COLOMBIA, 15 (38): 371-395 / JULIO-DICIEMBRE 2014 / 371

\section{El papel de la contabilidad ante la crisis financiera. Una reflexión sobre el conservadurismo incondicional de la NIIF 9*}

doi: 10.11144/Javeriana.cc15-38.pccf

\section{Ignacio de Cabo-Moreno}

Doctorando en Ciencias Económicas y Empresariales por

la Universidad de Córdoba, España. Profesor asociado

de la Universidad Loyola Andalucía, España.

Correo electrónico: icabo@uloyola.es

\section{Rafael Bautista-Mesa}

Doctor en Ciencias Económicas y Empresariales por la Universidad de Córdoba, España. Profesor de la Universidad Loyola Andalucía, España.

Correo electrónico: rbautista@uloyola.es

\author{
Horacio Molina-Sánchez \\ Doctor en Ciencias Económicas y Empresariales por la \\ Universidad de Córdoba, España. Profesor de la Universi- \\ dad Loyola Andalucía, España. \\ Correo electrónico: hmolina@uloyola.es

\section{Jesús N. Ramírez-Sobrino} \\ Doctor en Ciencias Económicas y Empresariales por la \\ Universidad de Córdoba, España. Profesor de la Universi- \\ dad Loyola Andalucía, España. \\ Correo electrónico: jramirez@uloyola.es
}

\footnotetext{
* Este documento corresponde a un artículo de reflexión. El artículo se deriva del proyecto de investigación SEJ425 de la Universidad Loyola Andalucía, España, cuyo título es La información financiera en la mejora de la competitividad de la empresa. Una versión preliminar de este artículo fue presentada en el XVI Congreso de la Asociación Española de Contabilidad y Administración de Empresas (AECA), celebrado en Granada (España), entre el 21 y el 23 de septiembre de 2011. Los autores agradecemos a los participantes en ese congreso sus valiosos comentarios.
} 
Resumen La Norma Internacional de Información Financiera 9 es la respuesta del International Accounting Standards Board - IASB - ante la perversidad del efecto procíclico de las políticas de reconocimiento del deterioro crediticio y ante la falta de objetividad que introducen en el modelo de reconocimiento los llamados trigger events - eventos disparadores-. La solución finalmente adoptada ha sido objeto de una significativa controversia durante el proceso de discusión. El trabajo realiza una revisión de las sucesivas propuestas tanto del IASB como del Financial Accounting Standards Board -FASB - estadounidense, y de las diferentes cartas de comentario recibidas por estos organismos, con el fin de sintetizar las diferentes alternativas estudiadas durante el proceso. El análisis pone de manifiesto que las consecuencias de la crisis financiera de 2008 han favorecido la evolución conceptual de la norma hacia el conservadurismo incondicional. Esta reforma es cuestionable con base en el Marco Conceptual, en especial en los primeros años de vida del activo financiero, pues puede suponer una merma en la representación fiel de la situación financiera de las empresas. Y, en cualquier caso, no exime al supervisor bancario de su intervención regulatoria en la búsqueda de la solvencia del sistema.

Palabras clave Deterioro crediticio; conservadurismo incondicional; crisis financiera; prociclicidad, pérdidas esperadas

\section{Clasificación JEL M41}

\section{The Role of Accounting before the Financial Crisis. A Reflection on the Unconditional Conservatism of the IFRS 9.}

\footnotetext{
Abstract The International Financial Reporting Standards 9 is the answer of the International Accounting Standards Board - IASB - before the perversity of the procyclical effect of the policies that acknowledge the credit impairment and the lack of objectivity that introduce in the acknowledgement model the so-called trigger events.
}

The solution that was finally adopted was subject to a significant controversy during the discussion process. This work makes a review of the successive proposals of the IASB, as well as those of the American Financial Accounting Standards Board - FASB — , and the different comment letters received by these entities, with the purpose of summarizing the different alternatives studied during the process. The analysis makes evident that the consequences of the financial crisis of 2008 have favored the conceptual evolution of the regulations towards unconditional conservatism. Based on the Conceptual Framework, this amendment is questionable, especially during the first years of the financial asset, as it might suppose a loss on the faithful representation of the financial situation of the companies. And, regardless of this, it does not free the bank supervisor from having to use his regulatory intervention, looking for the solvency of the system.

Keywords credit impairment; unconditional conservatism; financial crisis; procyclicity; expected losses

\section{Papel da contabilidade perante a crise financeira. Reflexão sobre o conservadorismo incondicional da NIIF 9}

Resumo A Norma Internacional de Informação Financeira 9 é a resposta do International Accounting Standards Board - IASB - ante a perversidade do efeito procíclico das políticas de reconhecimento da deterioração creditícia e ante a falta de objetividade que introduzem no modelo de reconhecimento os chamados de trigger events - eventos disparadores-. A solução finalmente adotada foi objeto de significativa controvérsia durante o processo de discussão. O trabalho realiza revisão das sucessivas propostas tanto do IASB quanto do Financial Accounting Standards Board -FASB - estadunidense, e das diferentes cartas de comentário recebidas por esses organismos, a fim de sintetizar as diferentes alternativas estudadas durante o processo. A análise põe de manifesto que as consequências da crise financeira de 2008 favoreceu a evolução concei- 
tual da norma para o conservadorismo incondicional. Essa reforma é questionável com base no Arcabouço Conceitual, em especial nos primeiros anos de vida do ativo financeiro, pois pode supor uma diminuição na representação fiel da situação financeira das empresas. E, em qualquer caso, não dispensa o supervisor bancário da sua intervenção regulatória na procura da solvência do sistema.

Palavras-chave Perdas creditícias; conservadorismo incondicional; crise financeira; prociclicidade; perdas esperadas

\section{Introducción}

La crisis de confianza en el sistema financiero, generada por la quiebra de entidades bancarias que meses antes gozaban de calificaciones crediticias elevadas, pone de manifiesto no solo las deficiencias de la supervisión bancaria internacional, sino también las deficiencias de una normativa contable que no genera información relevante sobre los instrumentos financieros y la actividad crediticia (Financial Stability Forum, 2008).

En el ámbito de la regulación bancaria, durante los últimos años, ha quedado patente la complejidad para preservar la estabilidad financiera internacional si las entidades financieras se gestionan con parámetros locales. El carácter global del sistema financiero trasciende en el Acuerdo Basilea III (Bank for International Settlements, BIS, 2010), al considerar un nuevo tipo de riesgo, el sistémico, que exige mayores ratios de capital y mayor liquidez a los activos financieros (en adelante, AF) que lo integran, para garantizar la estabilidad financiera (Goldin \& Vogel, 2010). En lo relativo a la gestión del riesgo de crédito, el enfoque de Basilea III se sigue basando en el concepto de pérdidas esperadas para el cálculo de los requerimientos de capital mínimo. Así pues, para la estimación del riesgo de insolvencia de los créditos concedidos se deben tener en cuenta no solo los eventos de impago efectivamente ocurridos, sino los posibles efectos derivados de incumplimientos futuros, bien mediante la consideración de calificaciones externas, bien mediante la aplicación de modelos internos.

En el ámbito internacional, los principales organismos reguladores están en proceso de homogeneización de las normas de información financiera mediante un proceso de armonización amparado en el acuerdo de convergencia o Memorandum of Understanding. Esta homogeneización, cuyo propósito es aumentar la comparabilidad de la información financiera internacional, contribuye a facilitar la supervisión del sistema bancario con carácter global, ya que este descansa sobre ratios cuya información de partida se encuentra en los estados financieros.

Por su significación en los estados financieros de las entidades de crédito, las normas relativas a los instrumentos financieros son especialmente relevantes en la determinación de la imagen fiel de su situación financiera (Borio \& Tsatsaronis, 2005). En este sentido, el International Accounting Standards Board (IASB) está modificando la normativa contable que regulaba los AF, la antigua Norma Internacional de Contabilidad 39 (NIC 39) y la está sustituyendo por la nueva Norma Internacional de Información Financiera 9 (NIIF 9), que pretende simplificar y homogeneizar la normativa existente al respecto. Dada su envergadura, este proyecto se ha dividido en tres fases. La primera 
de ellas se refiere a la clasificación y medida de todos los AF. La segunda fase busca determinar un modelo de reconocimiento del deterioro crediticio, mientras la tercera se dedica al registro de las operaciones de cobertura.

Nuestro trabajo se centra en la segunda fase, el reconocimiento del deterioro crediticio, y analiza el proceso de discusión seguido tanto por el IASB como por el FASB estadounidense, con el objetivo de contribuir a la reflexión acerca de si el conservadurismo incondicional ha de ser una característica básica de la información financiera como vía para asegurar una mayor garantía para acreedores e inversores, lo que ayuda al supervisor, en el caso de las entidades de crédito, en su búsqueda de la estabilidad del sistema financiero.

Durante el proceso de modificación de la NIC 39 se cuestionó cuáles deben ser los objetivos de la información financiera. El resultado hasta el momento, con evidentes influencias políticas, representa un giro hacia al conservadurismo incondicional como medida para la reducción del riesgo de futuras crisis financieras.

El FASB define el conservadurismo contable en el Statement of Financial Accounting Concepts -SFAC 2- al afirmar que "si en el futuro se van a producir unos cobros o pagos que tienen probabilidades similares de ocurrir, el conservadurismo consiste en usar la opción menos optimista”. Si ese conservadurismo se mide en términos condicionales o ex post, aplicado a la política de deterioro crediticio, se trata de reconocer las pérdidas no realizadas antes que los ingresos no realizados (Basu, 1997). El conservadurismo ex post implica una infravaloración condicional del resultado conta- ble en los períodos en que acontecen beneficios económicos (un incremento en la capacidad de generación de tesorería de la empresa), que se reconocerán cuando se realicen, mientras que las pérdidas económicas (la reducción en el valor actual de las expectativas de futuros flujos de tesorería de la empresa) serán incorporadas al resultado de forma oportuna.

El conservadurismo ex ante, derivado de los principios y normas contables, impide reconocer en el balance ciertos activos económicos (como la inversión en formación de personal), o incluso obliga a reconocer un menor valor de los activos, lo que motiva en ambos casos una subestimación incondicional o permanente del resultado contable. El conservadurismo incondicional, por tanto, establece políticas contables conservadoras en el tiempo (como la elección de un determinado modelo de reconocimiento del deterioro crediticio), mientras que el conservadurismo condicional está más relacionado con decisiones puntuales ante ingresos o valoraciones. Cabe esperar una relación inversa entre la prudencia ex ante y ex post, dado que cuanto menor sea el valor por el que se reconoce contablemente un activo, menor será la probabilidad de registrar una pérdida asociada a él.

El trabajo se estructura en cinco apartados. Tras la introducción, en el primer apartado señalamos las deficiencias del actual modelo de reconocimiento del deterioro crediticio y resaltamos las prácticas de manipulación contable relacionadas. El segundo apartado expone las diferentes propuestas de valoración del deterioro crediticio en la NIIF 9, marco necesario para el análisis en el tercer apartado de la evolución 
conceptual de la norma hacia el conservadurismo incondicional en sus diferentes borradores.

Mediante el análisis de las propuestas de los reguladores contables y de las diferentes cartas de comentarios remitidas por los diferentes usuarios de la información contable, valoramos los aspectos principales puestos de manifiesto a lo largo de la evolución de la norma. Como conclusión, coincidimos con la opinión de otros trabajos previos (Borio \& Tsatsaronis, 2004; Herz, 2010; Song, 2011, 2012), según los cuales los estados financieros de las entidades de crédito deben reflejar la imagen fiel de la empresa y servir de base homogénea y comparable sobre la que el supervisor bancario imponga el grado de prudencia necesario para garantizar la estabilidad del sistema financiero.

\section{Deficiencias en el modelo actual de reconocimiento del deterioro del crédito en la normativa internacional}

La política contable del reconocimiento del deterioro de los AF es de vital importancia para las empresas en las que los activos de este tipo tienen un peso significativo en sus balances, como las entidades financieras, las compañías aseguradoras y aquellas empresas con excedentes de liquidez que deciden invertir en deuda de terceros como vía para mejorar la rentabilidad.

Desde el momento en que se requieren estimaciones en el balance sobre las pérdidas futuras en los créditos, se abre paso a cierto grado de discrecionalidad que afecta la valoración de los créditos, el resultado del ejercicio y el capi- tal regulatorio exigido por el supervisor bancario, en el caso de las entidades de crédito.

El modelo de reconocimiento del deterioro crediticio actual, denominado de pérdidas incurridas, está basado en la existencia de una evidencia objetiva - trigger event-que determina el momento a partir del cual se hace necesario reconocer el deterioro crediticio en los estados financieros. Este modelo ignora la realidad del sistema financiero, que está consciente de que parte de los intereses generados por cada nuevo crédito concedido estarán destinados a cubrir futuras contingencias no solo de ese activo en concreto sino del resto de la cartera crediticia (Anagnostopoulos \& Buckland, 2005).

En la NIC 39 se detallan las diferentes evidencias objetivas que determinan si un activo o un grupo de activos están deteriorados; estos eventos deben ser observados por el tenedor del activo para el registro del correspondiente deterioro:

I. Dificultades financieras significativas del emisor o del obligado.

II. Incumplimientos de las cláusulas contractuales, tales como impagos o retrasos en el pago de los intereses o el principal.

III. El prestamista, por razones económicas o legales relacionadas con dificultades financieras del prestatario, otorga al prestatario, concesiones o ventajas que en otro caso no hubiera otorgado.

IV. Sea cada vez más probable que el prestatario entre en una situación concursal o en cualquier otra situación de reorganización financiera. 
V. La desaparición de un mercado activo para el activo financiero en cuestión, debido a dificultades financieras.

VI. Los datos observables indican que existe una disminución mensurable en los flujos de efectivo estimados futuros en un grupo de AF desde el reconocimiento inicial de aquellos, aunque la disminución no pueda ser todavía identificada con AF individuales del grupo (NIC 39.59).

En el caso de los AF contabilizados al coste amortizado, que constituyen la mayor parte de los AF de una entidad financiera basada en el negocio tradicional de intermediación, el deterioro reconocido es la diferencia entre el importe en libros del activo y el valor actual de los flujos de efectivo futuros estimados excluyendo las pérdidas crediticias futuras. El importe de la pérdida se reconoce en el resultado del ejercicio en el que se han detectado algunos de los eventos descritos con anterioridad, eventos que gozan de cierto grado de subjetividad (ver apartados I, IV, VI).

En períodos posteriores, si el importe de la pérdida por deterioro del valor disminuyese y esta pudiera ser objetivamente relacionada con un evento posterior al reconocimiento del deterioro (como la mejora en la calificación crediticia del deudor), la pérdida por deterioro reconocida previamente es objeto de reversión en el resultado del ejercicio. La reversión no da lugar a un importe en libros del activo financiero que exceda al coste amortizado que habría sido reconocido en la fecha de reversión si no se hubiese contabilizado la pérdida por deterioro del valor.

Según el IASB (2009a), el modelo de reconocimiento del deterioro crediticio actual presenta dos deficiencias básicas: (i) su carácter procíclico; y (ii) la discrecionalidad que entraña la verificación de los trigger events - eventos disparadores - El primer inconveniente radica en el carácter procíclico del modelo. Es decir, en épocas de crecimiento económico se obvian los potenciales riesgos futuros asociados a los AF generados, al no reconocer deterioro crediticio por las posibles pérdidas de las que aún no se tienen indicios, lo cual sobreestima los ingresos financieros; en épocas de recesión, por su parte, el deterioro reconocido crece de forma exponencial, lo que provoca una descapitalización del sistema financiero que obliga a restringir el crédito. La restricción del crédito genera un mayor deterioro de la actividad económica vía reducción de consumo y además un estancamiento de la inversión, que resulta relevante en aquellos países en los que la financiación ajena es esencialmente bancaria. Por el contrario, en los económicos expansivos, el deterioro reconocido es mínimo, lo que genera un mayor margen financiero, una mayor capitalización y por tanto, un mayor desarrollo del crédito (Albertazzi \& Gambacorta, 2009; Bikker \& Metzemakers, 2005; Dugan, 2009; González-Mota, 2005; Laeven \& Majnoni, 2003).

El segundo de los inconvenientes básicos es la discrecionalidad que la norma permite al preparador, al delegarle la capacidad de determinar algunos de los trigger events que dan lugar al reconocimiento del deterioro crediticio. Esta discrecionalidad otorga un margen de 
actuación al preparador en la formulación de las cuentas anuales que lo distancia de la fiabilidad de la información contable según la teoría de la asimetría de la información de George A. Akerloff (1970), ganador del premio Nobel de Economía en 2001, junto con Michael Spence y Joseph E. Stiglitz.

En la literatura contable, hay diferentes motivos que pueden inducir al preparador a manipular la información relacionada con el reconocimiento del deterioro crediticio como la gestión del capital, la valoración en los mercados financieros, la gestión de resultados y la optimización fiscal del resultado de la empresa (Blose, 2001). Estas prácticas distorsionan la imagen fiel de la situación financiera y de los resultados de las entidades, imagen que es el punto de partida para la regulación impuesta por el supervisor bancario.

La gestión de capital es clave en el ámbito de las entidades de crédito, pues sobre esta variable descansa el modelo de supervisión bancaria orientado a reconocer suficiente volumen de deterioro crediticio como para atender la totalidad de las pérdidas incurridas, de forma que no se produzca un consumo adicional de capital y la entidad financiera pueda hacer frente a pérdidas no esperadas vía recursos propios en caso de ser necesario. Por el contrario, si las pérdidas realizadas son mayores que las reconocidas, nos encontraremos con que se consumirá capital, lo que repercute en la capacidad financiera de la entidad, en sus resultados y en la proyección del valor de la empresa en el mercado (Wahlen, 1994). De ahí la importancia de la correcta capitalización de las entidades para asumir los escenarios posibles y el control ejercido por el supervisor bancario, sobre todo cuando la entidad haya desarrollado su propio sistema de gestión del riesgo.

Las instituciones financieras que mantienen un holgado margen sobre los requisitos mínimos de capital reciben financiación a un menor interés, no están seguidas de cerca por los organismos reguladores y tienen menos restricciones a la hora de materializar sus inversiones, motivo por el que los directivos tienden a alterar las políticas de reconocimiento del deterioro crediticio en beneficio propio (Deschênes, 2008; Lobo \& Yang, 2001).

La relación entre la gestión del capital y el reconocimiento del deterioro ha variado en el tiempo según han ido variando las exigencias establecidas por los acuerdos de Basilea (Bank for International Settlements, BIS, 1988, 2001, 2010) sobre el coeficiente de solvencia y la definición de capital regulatorio (Ahmed, Takeda \& Thomas, 1999; Anandarajan, Hasan \& LozanoVivas, 2003; Beatty, Chamberlain \& Magliolo, 1995; Kim \& Kross, 1998).

Dadas las significativas consecuencias de la política de reconocimiento de deterioro en las necesidades de capitalización de las entidades de crédito y su repercusión en sus niveles de actividad, el mercado reacciona positiva o negativamente cuando se producen cambios en la política de registro de deterioros (Ahmed, Takeda \& Thomas, 1999; Cortavarria, Dziobek, Kanaya \& Song, 2000; Kim \& Kross, 1998).

En general, en el reconocimiento del deterioro crediticio se puede diferenciar entre el reconocimiento por causas objetivas, contrastables y evidentes de impago, llamado deterioro objetivo, y en aquel que se reconoce de forma discrecional 
con base en criterios subjetivos, denominado deterioro discrecional (Wahlen, 1994). El mercado interpreta de forma positiva el reconocimiento del deterioro crediticio de forma discrecional pues considera que los directivos tienen confianza en los ingresos futuros de la empresa $y$, por ello, se permiten sacrificar beneficios actuales para compensar pérdidas futuras. El intento de los directivos por evitar la volatilidad del resultado es, en general, positivamente valorado por los inversores y acreedores, lo que debe provocar un aumento de la valoración de las acciones (Hartwig, 2013).

Por el contrario, si el reconocimiento del deterioro no discrecional, es interpretado como una corrección del valor real de los activos de la empresa que hace pensar que el valor de la empresa es relativamente menor que antes del anuncio del reconocimiento por lo que, en buena lógica, el precio de la acción debería bajar al despertar menor interés de los inversores (Beaver \& Engel, 1996; Blose, 2001). En España, María José Arcas-Pellicer (1994) encuentra evidencias de que la publicación de un resultado por debajo de las expectativas del mercado provoca, aunque no de forma inmediata, un ajuste en el precio de cotización de las acciones.

La relación existente entre el precio de la acción y el deterioro crediticio es más significativa, si la ratio de capital de la empresa está ajustada al mínimo, ya que un incremento del volumen de AF deteriorados limitaría en exceso la capacidad financiera de la empresa, lo cual podría situarla bajo la vigilancia de los supervisores que podrían sugerir la intervención o estatalización de la entidad (Kanagaretnam, Lobo \& Yang, 2005).
Otro incentivo habitual para la manipulación del resultado vía deterioro crediticio, es el alisamiento del beneficio. Esta práctica consiste en sobreponderar el reconocimiento de deterioro en períodos de expansión económica y reducir las provisiones en épocas de recesión. La gestión de la cuenta de resultados vía reconocimiento del deterioro crediticio es una práctica habitual, porque no conlleva alteración de los flujos de caja, reduce el pago de impuestos en épocas de bonanza y, al no acarrear consecuencias sobre los flujos de efectivo, es difícilmente detectable por los reguladores (Dechow \& Skinner, 2000). La discrecionalidad de la actual NIC 39 permite a los directivos reconocer pérdidas que disminuyan la carga fiscal global de la empresa. Si la política de reconocimiento es estructuralmente conservadora, el flujo de los pagos a hacienda disminuirá en el tiempo, lo que proyecta, adicionalmente, un mayor valor de la empresa (Watts, 2003a; Qiang, 2007).

El alisamiento del beneficio es una práctica relacionada con menores costes de financiación (Dechow, Sloan \& Sweeney, 1996; Petersen \& Thiagarajan, 2000), puesto que las empresas con baja volatilidad en sus resultados presentan una menor prima de riesgo (Gebhardt, Reichardt \& Wittenbrink, 2004). Por último, las empresas con alto volumen de beneficios despiertan el interés tanto del supervisor como de los diferentes grupos de presión (consumidores, empleados, etc.). Los directivos hacen uso discrecional del reconocimiento del deterioro para reducir su visibilidad ante el supervisor y los auditores (Liu \& Ryan, 2006; Watts \& Zimmerman, 1983) y evitar así que los reguladores fijen nuevos impuestos, inicien investigaciones antimonopolio, reduzcan 
las ayudas al sector o modifiquen los precios en el caso de que estos estén intervenidos.

Para combatir las deficiencias de la NIC 39, la investigación existente destaca algunas herramientas como el desarrollo de normas contables de calidad (Fonseca \& González, 2008), el desarrollo de regulación que proteja al inversor (Shen \& Chih, 2005), la supervisión bancaria (Pérez, Salas-Fumás \& Saurina, 2008) y el desarrollo de sistemas retributivos a los directivos no ligados al precio de las acciones (Cornett, McNutt \& Tehranian, 2009).

\section{Evolución del modelo de reconocimiento del deterioro crediticio en la NIIF 9}

Independientemente de la tradición contable, la regulación vigente sobre AF se ha ido modificando en el tiempo al ritmo que la realidad regulada, el mercado financiero, ha ido evolu- cionando. Estas modificaciones hacen que la norma sea prolija (tabla 1), compleja en cuanto a su seguimiento y, en algunos puntos, arbitraria en cuanto a su implementación (IASB \& FASB, 2008). De ahí que uno de los criterios básicos en el desarrollo de las modificaciones sea el de su simplificación para facilitar no solo el cumplimiento de la norma sino el uso de la información generada relacionada con los AF.

Aunque con la intención de aunar esfuerzos en la modificación de la NIC 39, reflejada en la emisión conjunta de un borrador común para la norma de AF (IASB \& FASB, 2008), los itinerarios seguidos por el IASB y el FASB son diferentes. El IASB está inmerso en un modelo de desarrollo de la norma en tres fases y el FASB propone la reforma de la norma como algo integral. La tabla 2 recoge los diferentes documentos o borradores emitidos al respecto por ambos reguladores desde el inicio del procedimiento de modificación de la norma hasta la fecha.

\section{IASB}

FASB

\begin{tabular}{ll}
\hline - NIIF 7 Financial Instruments: Disclosures. & - SFAS 65 Accounting for Certain Mortgage Banking Activities. \\
- NIC 32 Financial Instruments: Presentation. & - SFAS 107 Disclosures about Fair Value of Financial Instruments. \\
- NIC 39 Financial Instruments: Recognition & - SFAS 114 Accounting by Creditors for Impairment of a Loan. \\
and Measurement. & - SFAS 115 Accounting for Certain Investments in Debt and Equity Securities. \\
& - SFAS 133 Accounting for Derivative Instruments and Hedging Activities. \\
& - SFAS 140 Accounting for Transfers and Servicing of Financial Assets and \\
& Extinguishments of Liabilities. \\
& - SFAS 150 Accounting for Certain Financial Instruments with Characteristics \\
& of both Liabilities and Equity. \\
& - SFAS 155 Accounting for Certain Hybrid Financial Instruments. \\
& - SFAS 157 Fair Value Measurements. \\
& - SFAS 159 The Fair Value option for Financial Assets and Financial \\
& Liabilities. \\
\hline
\end{tabular}

Tabla 1. Normativa internacional sobre AF - Reconocimiento del deterioro crediticio

Fuente: elaboración propia, a partir de información contenida en el Discussion Paper (DP), emitido en marzo 2008 por IASB y FASB. 


\begin{tabular}{|c|c|c|}
\hline Fecha & Documento & Título \\
\hline Marzo de 2008 & Discussion Paper & $\begin{array}{l}\text { Reducing Complexity in Reporting Financial Instruments (IASB } \\
+ \text { FASB) }\end{array}$ \\
\hline Junio de 2009 & Request for Information & $\begin{array}{l}\text { Impairment of Financial Assets: Expected Cash Flow Approach } \\
\text { (IASB) }\end{array}$ \\
\hline Noviembre de 2009 & Exposure Draft & Financial Instrument: Amortised Cost and Impairment (IASB) \\
\hline Mayo de 2010 & Exposure Draft & $\begin{array}{l}\text { Accounting for Financial Instruments and Revisions to the } \\
\text { Accounting for Derivative Instruments and Hedging Activities } \\
\text { (FASB) }\end{array}$ \\
\hline Enero de 2011 & $\begin{array}{l}\text { Supplement to Exposure } \\
\text { Draft } 11 / 2009\end{array}$ & $\begin{array}{l}\text { Joint Proposed Approach to Accounting for Credit Losses } \\
\text { (IASB+FASB) }\end{array}$ \\
\hline Diciembre de 2012 & Exposure Draft & $\begin{array}{l}\text { Proposed Accounting Standards Update Financial Instruments - } \\
\text { Credit Losses (FASB) }\end{array}$ \\
\hline Marzo de 2013 & Exposure Draft & Financial Instruments: expected Credit Losses (IASB) \\
\hline
\end{tabular}

Tabla 2. Cronograma documental de la creación de la NIIF 9

Fuente: elaboración propia

Conocedores de las deficiencias de la normativa actual que regula los $\mathrm{AF}$, en marzo de 2008, el IASB y el FASB comenzaron el proceso de reforma de la normativa con la emisión de un discussion paper (IASB \& FASB, 2008) que aboga por un único criterio contable, el valor razonable - fair value - para valorar todos los AF. El valor razonable, entendido como valor de mercado o valor de salida de un AF, permite reconocer como deterioro las pérdidas anticipadas por el mercado. Así mismo, elimina la subjetividad del análisis del trigger event necesario bajo el modelo de pérdidas incurridas.

La elección del valor razonable por el IASB y el FASB como único método de valoración está marcada por la incapacidad del modelo de coste amortizado para proporcionar información relevante y útil de aquellos AF cuyo valor no está necesariamente relacionado con los flujos de caja que generan. Según ambos Consejos, el valor razonable permitiría incorporar en la información contable proyecciones futuras, in- cluidas las pérdidas esperadas por el mercado, lo que elimina uno de los inconvenientes del modelo de pérdidas incurridas (IASB \& FASB, 2008, párrafo 3.24).

Los preparadores financieros, los supervisores bancarios y los usuarios son los que indican que esa homogeneización provoca una elevada volatilidad en las cuentas de resultados y no refleja la realidad de una parte importante de los $\mathrm{AF}$, que son los $\mathrm{AF}$ valorados a coste amortizado. Este modelo de valoración no contribuye a reflejar la imagen fiel de unos activos que se recuperarán por el retorno de los flujos contractuales pactados y no por su venta $y$, por tanto, no se deben ver afectados por las fluctuaciones de valor en el mercado. El Comité de Basilea se posiciona en este sentido: “...Estamos de acuerdo en que el valor razonable es apropiado para las operaciones de negociación, en las cuales los flujos de efectivo se generan por la compra y venta de los activos, algo que presupone la existencia de 
mercados. Sin embargo, creemos que el sentido de las operaciones de captación de depósitos y concesión de préstamos de la banca convencional están mejor representadas por el enfoque actual del coste amortizado. Aquí los flujos de caja se generan normalmente por el diferencial entre tasas activas y pasivas $y$, en general, no dependen del valor razonable de estos instrumentos, puesto que no son negociados..." (CL\#94, ${ }^{1}$ Comité de Basilea).

Así mismo, entre los inconvenientes de aplicar un modelo de valor razonable sobre los $\mathrm{AF}$, se encuentran las fluctuaciones que pueden generar las valoraciones de los AF en los mercados financieros que se ven afectados por numerosas variables y que, en determinadas circunstancias, gozan de cierta iliquidez para AF singulares (Allen \& Carletti, 2008; Boyer, 2007; Hellwig, 2009; Kothari \& Lester, 2012; Matherat, 2008; Persaud, 2008; Plantin, Sapra \& Shin, 2008).

Tras la recepción de numerosas críticas por proponer un método de valoración a precios de mercado para AF no destinados a la venta (CL\#22 International Banking Federation), el IASB propone aplicar valor razonable para aquellos AF mantenidos para la venta y un modelo alternativo para los AF que en la NIC 39 están valorados a coste amortizado: el modelo de pérdidas estimadas propuesto por el IASB en junio de 2009 (IASB, 2009a) y reafirmado en noviembre de 2009 (IASB, 2009b).

1 Carta de comentario al Discussion Paper. Reducing Complexity in Reporting Financial Instruments, emitido por IASB\&FASB en marzo de 2008.

2 Carta de comentario al Discussion Paper, Reducing Complexity in Reporting Financial Instruments, emitido por IASB\&FASB en marzo de 2008
Según este modelo de reconocimiento del deterioro, el valor contable de los AF está relacionado directamente con los flujos de caja que generan, incluyendo en estos flujos las pérdidas estimadas. Este modelo supone un cambio importante en la normativa contable en la medida en que permite recoger predicciones en los estados financieros al reconocer en las cuentas de resultados pérdidas no realizadas y sobre las cuales aún no se tiene evidencia, lo que disminuye el valor de los AF en balance. Según este modelo, el valor contable de los AF es el valor actual de los flujos de caja estimados descontados al tipo de interés efectivo de la operación. El tipo de interés efectivo se calcula distribuyendo los flujos de caja contractuales teniendo en cuenta además las pérdidas estimadas que se pueden producir a lo largo de la vida del AF.

El modelo de flujo de pérdidas estimadas tiene el objetivo de recoger las dos dimensiones de los AF valorados a coste amortizado, la generación de flujos de caja futuros y la asunción de riesgo. Sus características principales son: (i) los ingresos financieros se reconocen con base en los flujos de caja esperados incluyendo la probabilidad de impago; (ii) el deterioro se produce como un cambio negativo en las expectativas de crédito; (iii) el deterioro de crédito se reconoce en la cuenta de resultados como la diferencia del valor del activo en balance y los flujos de caja estimados actualizados; (iv) para actualizar los flujos de caja se usa el tipo de interés efectivo; el deterioro es reversible en la cuenta de resultados cuando hay un cambio positivo en las expectativas de cobro. 
Entre los inconvenientes de este modelo está el incremento del coste de preparación, no solo para las entidades financieras, al obligar a realizar complejas estimaciones sobre las pérdidas futuras. Las entidades financieras, unos de los principales afectados por esta reforma, señalan además que la información resultante no sería acorde con los modelos de gestión de riesgo seguidos por las entidades financieras que no están bajo el modelo de Internal Rating Based (CL \#148 ${ }^{3}$ Comité de Basilea).

Para minimizar el coste de implementación del modelo, el IASB propone inicialmente recurrir a un modelo que tenga como base de cálculo del deterioro crediticio las tasas históricas de impago de AF de similares características. El sistema propuesto sigue generando rechazo entre los usuarios de la información financiera, las entidades financieras, los supervisores bancarios e incluso el FASB, por lo que el IASB decidió en diciembre de 2009 crear un panel de expertos (Expert Advisory Panel, EAP), para analizar las carencias del modelo y proponer alternativas para mejorar el criterio de valoración. De la intervención directa de los agentes interesados por medio del due process, e indirecta mediante su presencia en el EAP, nacieron diferentes propuestas acerca de cómo calcular las pérdidas estimadas, cuya evolución se muestra esquemáticamente en la ilustración 1.

En mayo de 2010, el FASB emite un Proposed Accounting Standards Update (PASU) (FASB, 2010), que propone un único modelo para reconocer y valorar el deterioro crediticio

3 Carta de comentario al Exposure Draft. Financial Instruments: Amortised Cost and Impairment, emitido por el IASB en diciembre de 2009. de todos los AF. En el PASU, el FASB propone reconocer en la fecha de cierre la totalidad del deterioro estimado en su cartera crediticia para generar reservas suficientes que puedan paliar las pérdidas incurridas y esperadas. En lo que se refiere a las pérdidas estimadas, este modelo está totalmente alineado con el que demanda el Comité de Basilea en su carta de comentario remitida a ambos organismos el 1 de septiembre de 2009, en la que indica que a fecha de cierre se deben reconocer tantas pérdidas como se estimen para el resto de la vida de la cartera de activos y aclara en la memoria los motivos que llevan a reconocer tales deterioros.

Meses más tarde, en enero de 2011, ambos reguladores emitieron un documento conjunto (IASB \& FASB, 2011), complementario al borrador de noviembre de 2009 emitido por el IASB, en el que siguieron reflexionando principalmente sobre la importante decisión de o bien reconocer el deterioro total estimado en el momento de la concepción del AF (FASB) o diferirlo a lo largo de la vida útil del AF, mediante el uso de modelos intermedios (IASB).

El supervisor bancario propone entonces un modelo intermedio que usa la tasa de pérdidas (Probability of Default, PD) estimadas para cada cartera de AF en el cálculo de las pérdidas estimadas al igual que hacen en la actualidad las entidades financieras para cumplir los requisitos de Basilea. El volumen de deterioro reconocido sería suficiente para hacer frente a las pérdidas que se van realizando año a año para este tipo de AF. Esa tasa de pérdidas es conocida y se toma de los modelos de riesgo de cada una de las entidades, lo que facilita su aplicación a efectos de información financiera. 
LA CONTABILIDAD ANTE LA CRISIS FINANCIERA / I. DE CABo, R. BAUtista, H. MOLINA, J. RAMíREZ / 383

\begin{tabular}{lll}
\hline Fecha & \multicolumn{1}{c}{ Organismo } & \multicolumn{1}{c}{ Modelo } \\
\hline & & Valor razonable \\
$3 / 2008$ & IASB + FASB & \\
& & Modelo de pérdidas estimadas \\
\hline $6 / 2009$ & IASB & Modelo de pérdidas estimadas \\
\hline $11 / 2009$ & IASB & Pérdidas lineales \\
\hline $2 / 2010$ & IASB (Expert Advisory Panel) \\
\hline $3 / 2010$ & IASB (Expert Advisory Panel) & Pérdidas históricas \\
\hline $4 / 2010$ & IASB (Expert Advisory Panel) & Modelo de convergencia - pérdidas lineales \\
\hline $11 / 2010$ & IASB + FASB (Board Meeting) & Modelo de convergencia (lineal, histórico, libro medio) \\
\hline $12 / 2010$ & IASB + FASB (Board Mee- & Modelo de convergencia (lineal, histórico, libro medio) \\
& ting) & y de emergencia \\
\hline $1 / 2011$ & IASB + FASB & Modelo de convergencia (Basilea, futuro predecible) \\
\hline $6 / 2011$ & IASB + FASB (Board Meeting) & El modelo "The Buckets" \\
\hline $12 / 2012$ & FASB & Modelo de pérdidas probables \\
\hline $3 / 2013$ & IASB & Modelo “Three Stages" \\
\hline
\end{tabular}

Ilustración 1. Evolución conceptual de la NIIF 9

Fuente: elaboración propia

Además, propone definir un porcentaje mínimo de deterioro a reconocer en aquellas carteras de AF cuya tasa histórica sea pequeña o nula con la intención de generar provisiones suficientes para afrontar pérdidas probables en caso de un cambio de ciclo. Este porcentaje mínimo se asemeja a la provisión dinámica española, comúnmente llamada en la literatura provisión anticíclica o provisión genérica, según la Circular 4/2004 del Banco de España.

Como acercamiento a la proposición del regulador bancario, el EAP elabora un segundo modelo que cuenta con el beneplácito de los reguladores contables, del Comité de Basilea y del organismo que representa a la banca europea: el modelo de convergencia.

Este modelo propone acercar la gestión que las entidades hacen de su cartera crediticia a los estados financieros mediante la creación de un libro de AF buenos y un libro de AF malos (good book-bad book). El primero está compuesto por AF que no se prevé que vayan a deteriorarse en el siguiente ejercicio contable. El segundo libro estará formado por AF deteriorados y por aquellos cuya expectativa de impago es elevada.

En un acercamiento claro al modelo de reconocimiento propuesto por el FASB (2010), el EAP propone aplicar esta metodología a los AF ubicados en el libro malo. Sin embargo, para los AF ubicados en el libro bueno cada año se reconocería el deterioro anual medio previsto para la vida útil de la cartera, con un mínimo igual a las pérdidas esperadas en los doce meses siguientes. En el caso de que un AF ubicado en el libro bueno resultara impagado, se trasladaría al 
libro malo, se traspasaría en ese momento el total del deterioro reconocido de un libro al otro y se reconocería todo el deterioro previsto para ese AF. Otra propuesta barajada para el reconocimiento del deterioro en los AF ubicados en el libro bueno es aplicar la probabilidad de impago (PD), calculada por el modelo de riesgos de Basilea, sobre la cartera para calcular el deterioro total esperado para después prorratearlo linealmente en función de la edad media de los AF que componen la cartera. Este modelo lo hemos llamado modelo de convergencia de pérdidas lineales.

Otro modelo entre los convergentes es el modelo de tasas históricas que, en lugar de aplicar PD, aplica una tasa histórica de pérdidas según carteras de AF. Este modelo requiere realizar subcarteras dentro del libro bueno en las que se ubiquen AF con tasas históricas de impago similares.

En una propuesta cada vez más cercana al FASB, el IASB propone la creación de un libro medio en el que se ubicarían aquellos AF al corriente de pago pero con una alta probabilidad de deterioro y medidos en términos de cartera. En el libro medio, al igual que en el libro malo, se reconocería todo el deterioro esperado, pero el seguimiento del activo se haría en términos de cartera mientras que en el libro malo se haría de forma individual. Este modelo, al que hemos denominado modelo del libro medio, es una variación más conservadora que permite clasificar los AF en: "al corriente de pago", "de dudoso cobro" e "impagados". Al mismo tiempo surgen otras cuatro formas de cálculo de las pérdidas estimadas: el modelo de Basilea, el modelo de emer- gencia y los modelos de futuro predecible con y sin actualización de flujos de caja.

El modelo de Basilea propone aplicar una tasa de pérdidas mínimas sobre los AF ubicados en el libro bueno, mientras que el modelo de período de emergencia propone definir un período de entre dos y tres años para calcular las pérdidas estimadas en ese tiempo, denominado período de emergencia. En el cierre del ejercicio se reconocería todo el deterioro estimado para ese período. De esta forma, se pretende que la institución financiera genere provisiones suficientes para mantener la actividad durante el período de emergencia.

El modelo de futuro predecible propone reconocer como deterioro crediticio el deterioro estimado para la cartera de activos en un período en el que las estimaciones sean consistentes, actualizando el valor de los flujos de caja previstos. En el caso de no actualización de los flujos surge el tercer modelo, el modelo de convergencia de futuro predecible sin actualización.

En enero de 2011, en un borrador, ambos reguladores proponen reconocer todo el deterioro esperado de los AF ubicados en el libro malo y el mayor deterioro resultante de los siguientes cálculos para los AF ubicados en el libro bueno: (a) la pérdida anual media esperada. Es decir, se calcula el deterioro total de la cartera hasta vencimiento y se divide por la vida media de los AF en la cartera; o (b) la pérdida que se puede calcular con precisión en un futuro inmediato que, como mínimo, será de un año.

Casi dos años más tarde, el FASB en solitario emitió una actualización de proyecto de reforma de la norma de reconocimiento del 
deterioro crediticio, Proposed Accounting Standards Update: Financial Instruments-Credit Losses, en la que obliga a reconocer todo el deterioro crediticio esperado calculado teniendo en cuenta la información histórica de la empresa, las circunstancias actuales y las estimaciones que la empresa considere razonables con base en sus modelos internos.

El último paso dado por el IASB es la publicación del documento Financial Instruments: Expected Credit Losses, en el que se establece un nuevo modelo, el modelo de tres fases. Este modelo propone que en una fase inicial, en la que se genera el activo financiero, se reconozca el deterioro esperado para los primeros doce meses de vida del AF. En una segunda fase, si la calidad crediticia del activo financiero se viese alterada de forma drástica, se reconocerían todas las pérdidas esperadas para ese activo pero se seguiría incluyendo la totalidad del interés generado en la cuenta de resultados. En una tercera fase, si el activo resulta impagado, se reconocerá la totalidad del deterioro esperado y solo se considerarán los intereses calculados sobre el valor residual del AF, considerado este como la diferencia entre el valor del AF y el deterioro esperado.

De nuevo, el Comité de Basilea presiona hacia un mayor conservadurismo en su carta de comentario del 21 de junio de 2013 al IASB, en la que insta a reconocer mayor deterioro crediticio en la fase inicial para garantizar que se genera un colchón suficiente para amortiguar las pérdidas que pudiera generar el peor de los escenarios económicos en un año, e indica que sería el punto de partida para una normativa contable internacional homogénea en cuanto al reconocimiento del deterioro crediticio se refiere.
A modo de resumen, la tabla 3 indica de forma esquemática los diferentes modelos antes mencionados.

\section{El conservadurismo en el marco conceptual del IASB y en la reforma del deterioro crediticio}

El proceso de convergencia de los criterios de reconocimiento del deterioro crediticio entre los modelos del IASB y el FASB, está marcado por una tendencia contable hacia el conservadurismo incondicional, como elemento clave para evitar futuras crisis financieras. La propuesta ha evolucionado desde el modelo del valor razonable hasta una valoración de los AF más conservadora, mediante el reconocimiento del deterioro crediticio esperado desde el registro inicial del AF, como se aprecia de forma esquemática en la ilustración 2 .

Tanto el IASB como el FASB responden así a la demanda del $\mathrm{G} 20^{4}$ de introducir mayor prudencia a la hora de reconocer el valor de los AF en la información contable, herramienta básica para paliar el uso del conservadurismo condicional que origina la manipulación contable que permite alisar el beneficio, gestionar el capital o que los directivos envíen señales al mercado en su propio beneficio (Blose, 2001).

\footnotetext{
4 El Grupo de los Veinte (G20) reúne a los líderes de las principales economías desarrolladas. Los miembros del G20 representan el 85\% del producto bruto interno mundial, más del 75\% del comercio mundial y dos tercios de la población del mundo. En 2014, los miembros son Alemania, Arabia Saudita, Argentina, Australia, Brasil, Canadá, China, Corea del Sur, Estados Unidos, Francia, India, Indonesia, Italia, Japón, México, Reino Unido, Rusia, Sudáfrica, Turquía y la Unión Europea.
} 


\begin{tabular}{ll}
\hline \multicolumn{1}{c}{ Modelos } & \multicolumn{1}{c}{ Definición } \\
\hline $\begin{array}{l}\text { 1. Modelo } \\
\text { de pérdidas }\end{array}$ & $\begin{array}{l}\text { En vigor en la NIC 39, para los instrumentos medidos a coste amortizado, solo permite reconocer } \\
\text { deterioro crediticio incurrido si hay una evidencia objetiva o trigger event que determine que el AF está } \\
\text { incurridas }\end{array}$ \\
\hline $\begin{array}{l}\text { deteriorado. } \\
\text { valor razonable de }\end{array}$ & $\begin{array}{l}\text { El valor razonable permite reconocer las pérdidas esperadas por el mercado para anticiparse a su } \\
\text { desencadenante. }\end{array}$ \\
\hline
\end{tabular}

El deterioro se reconoce distribuyendo la pérdida total estimada durante la vida útil del AF.

3.1 Basilea: se calcula bien mediante una tasa histórica de pérdidas estimadas o bien mediante el uso de un porcentaje de pérdidas.

3.2 De convergencia: creación de un libro bueno y de un libro malo de AF. El primero está compuesto de AF que no se prevé vayan a deteriorarse en el siguiente ejercicio contable. El segundo libro estará formado por AF impagados y por aquellos cuya expectativa de impago es alta. Propone reconocer todo el deterioro en el libro malo y aplicar el modelo de coste amortizado para el libro bueno. Según el modelo de cálculo aplicado sobre los AF ubicados en el libro bueno para reconocer el deterioro a cierre del ejercicio, los modelos de convergencia se pueden clasificar de la siguiente forma:

3.2.1 Pérdidas lineales: se distribuye la pérdida estimada en función de la vida útil de los activos de la cartera.

3.2.2 Tasa de pérdidas histórica: se aplica una tasa histórica de pérdidas para el cálculo del deterioro desde el reconocimiento inicial del AF.

3.2.3 Libro medio: creación de un libro intermedio que contendría AF al corriente de pago pero con probabilidad de deterioro alta; en él se reconocería igualmente todo el deterioro previsto en el momento inicial.

3.2.4 Basilea: propone aplicar una tasa de pérdidas mínimas sobre los AF ubicados en el libro bueno. 3.2.5 Período de emergencia: define un período de entre dos y tres años para calcular las pérdidas

3. Modelo de pérdidas estimadas estimadas en ese tiempo.

3.2.6 Futuro predecible: se reconoce el mayor deterioro: o el previsto para el próximo año o el resultado de dividir las pérdidas totales esperadas entre la vida útil media de la cartera de activos actualizada.

3.2.7 Futuro predecible sin actualización: igual que la opción 3.2.6, permite la no actualización del prorrateo de las pérdidas estimadas.

3.3 Pérdidas lineales: se calcula el deterioro como las pérdidas totales prorrateadas en función de la vida media de los AF de la cartera y se actualizan los flujos calculados al tipo de interés para AF libres de riesgo.

3.4 Pérdidas históricas: se calcula el deterioro en función de las tasas de deterioro históricas, se ubican en el tiempo y se actualizan al tipo de interés efectivo.

3.5 Conservador: se aplica una tasa histórica de pérdidas a la cartera de AF y se reconoce el deterioro previsto en el momento de concepción o compra del AF. Si no hay tasas históricas o fuesen irrelevantes, se aplica un porcentaje mínimo prudente.

3.6 De emergencia: se define un período de entre dos y tres años a contar desde la fecha de cierre para calcular las pérdidas estimadas en ese tiempo, denominado período de emergencia.

3.7 Tres fases: al originar el AF se reconoce el deterioro esperado para los doce primeros meses. Si hay un descenso significativo en la calidad del AF, se reconoce todo el deterioro crediticio estimado pero se sigue reconociendo la totalidad de los intereses generados. En el caso de pérdida incurrida también se reconoce la totalidad del deterioro estimado pero solo se trasladan a resultados los intereses generados por el valor neto del activo financiero.

3.8. Modelo FASB: se reconoce todo el deterioro crediticio esperado a cierre del ejercicio a partir de información histórica y actual. El deterioro es reversible.

Tabla 3. Modelos de reconocimiento del deterioro crediticio en el desarrollo de la NIIF 9

Fuente: elaboración propia 
LA CONTABILIDAD ANTE LA CRISIS FINANCIERA / I. DE CABo, R. BAUTISTA, H. MOLINA, J. RAMíREZ / 387

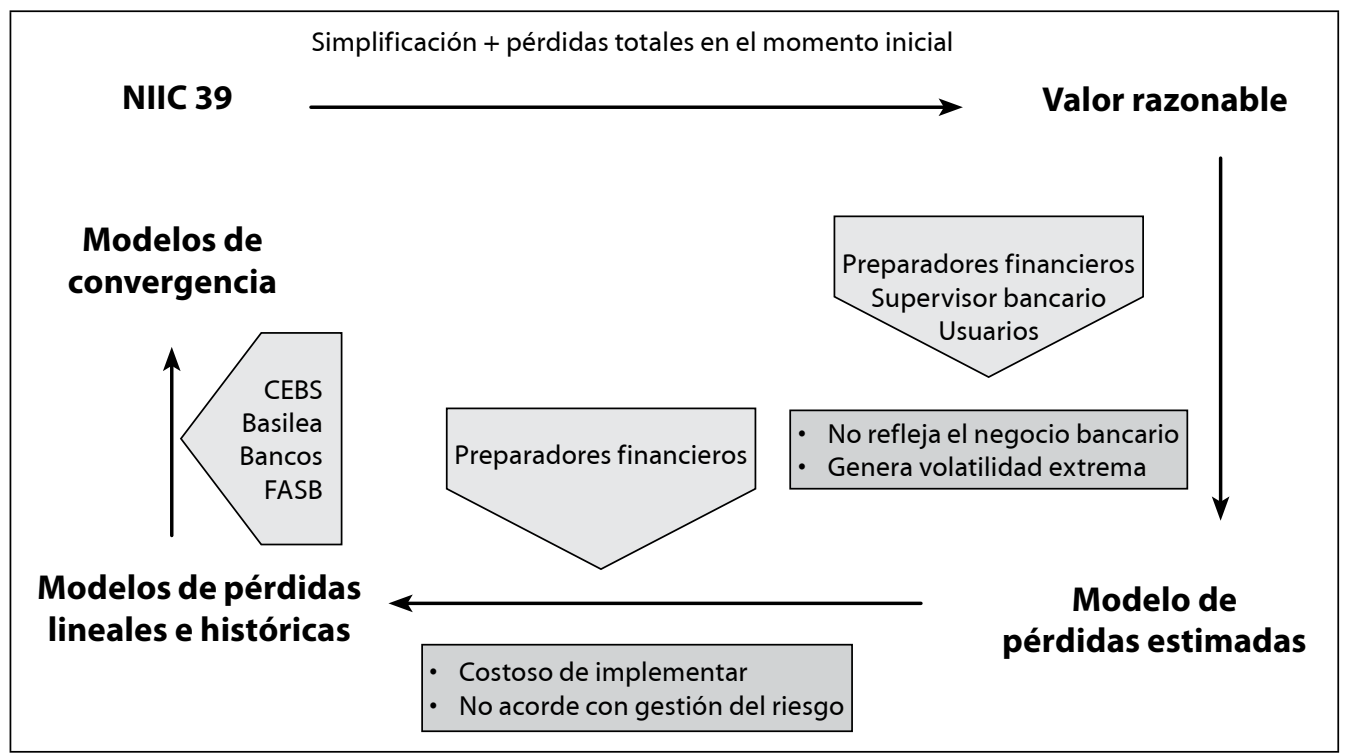

Ilustración 2. Evolución de los modelos propuestos en el desarrollo de la NIIF 9

Fuente: elaboración propia

El cambio regulatorio propuesto introduce una visión del concepto de AF influenciado por el conservadurismo incondicional, ya que recoge estimaciones futuras de las pérdidas que probablemente experimente el activo crediticio y que tienen como resultado una información financiera más prudente en lo que a reconocimiento del riesgo se refiere, lo que genera menor volatilidad en los flujos de caja y valora los recursos propios con mayor cautela. Esta prudencia en el reconocimiento del valor de los AF, como recogen Manuel Cano-Rodríguez (2010), Juan Manuel García-Lara, Beatriz García-Osma y Fernando Peñalva (2009, 2011) y Juan Antonio Rueda-Torres (2006), tiene como objeto proteger los derechos de los acreedores, de los inversores y de los depositantes de las entidades financieras; esto vuelve más eficientes los contratos entre estas partes.
Según la literatura existente, el conservadurismo incondicional es clave no solo porque beneficia a los usuarios de la información contable sino también porque mitiga el efecto contagioso en el mercado financiero de las quiebras de las entidades financieras (Biddle, Ma \& Wu, 2012). En este sentido, se observa que cierto grado de conservadurismo incondicional en las normas contables disminuye la probabilidad de quiebra de las entidades, con lo cual favorece la ansiada estabilidad financiera (Basu, 1997; Francis, Hasan \& Wu, 2013; Mohammed, Ahmed \& Ji, 2010). Por último y también a favor del conservadurismo incondicional, el hecho de que los bancos más conservadores reducen en menor medida su actividad crediticia en períodos de recesión, punto este de vital importancia para el desarrollo económico de una jurisdicción (Watts, 2003b). 
En el Marco Conceptual del IASB (2010), la prudencia no es una característica fundamental de la información financiera, ni tan siquiera una característica de mejora de la utilidad de los estados financieros. El concepto de prudencia sí permanece, aunque de forma implícita, en el reconocimiento inmediato de los gastos (IASB, 2010, párrafo 4.52) o en el necesario ejercicio de un cierto grado de precaución al realizar las estimaciones requeridas bajo condiciones de incertidumbre, de manera que los activos o los ingresos no se sobrevaloren, y que los pasivos o los gastos no se infravaloren. Así pues, el ejercicio de la prudencia no permite la creación de reservas ocultas o provisiones excesivas, la minusvaloración deliberada de activos o ingresos, ni la sobrevaloración consciente de pasivos o gastos, porque de lo contrario los estados financieros no resultarían fiables.

Por tanto, los fines prudenciales regulatorios son expresamente eliminados de los objetivos de la información financiera, cuyo objetivo es proveer la información útil a inversores y acreedores para la toma de decisiones sobre la aportación de recursos a una empresa (IASB, 2010, párrafo OB2). El IASB indica expresamente en su Marco Conceptual que los estados financieros de propósito general no están dirigidos principalmente a otras partes interesadas, como los reguladores, aunque reconoce que estos podrían encontrarlos útiles para sus propósitos (IASB, 2010, párrafo OB10).

Por este motivo, la introducción de un modelo de pérdidas esperadas puede representar, en algún sentido, una contradicción con los objetivos principales de la información financiera. Los acontecimientos derivados de la crisis financiera de 2008 aceleraron la resolución de esta discusión conceptual al "revelar significativas debilidades del modelo de pérdidas incurridas de la NIC 39" (IASB, 2009a, p. 4).

La justificación para la adopción de un modelo que anticipaba pérdidas de crédito antes del efectivo suceso de impago puede ser, en nuestra opinión, congruente con el actual Marco Conceptual. Según el IASB, el momento en que la pérdida de crédito tiene lugar no siempre es conocido pero, en todo caso, parece claro que el trigger event en el modelo de pérdidas incurridas retrasa el reconocimiento de pérdidas probables (IASB, 2009a, párrafo 5).

Sin embargo, el reconocimiento de una pérdida esperada durante los primeros meses de vida del crédito es, en nuestra opinión, más discutible, puesto que el riesgo de impago está implícitamente registrado en el coste inicialmente negociado por el activo. En este sentido, el propio IASB reconoce que el registro de un deterioro igual a una cantidad de " 12 meses de pérdidas esperadas en la etapa 1 no tiene justificación conceptual", pero supone una buena solución en términos coste-beneficio, así como una buena aproximación para ajustar los ingresos por intereses a un posible deterioro no significativo de la calidad crediticia durante ese período (IASB, 2013b, párrafo 7).

\section{Conclusiones}

Como respuesta a la demanda de mayor prudencia a la hora de reconocer el valor de los AF, y entre las diferentes alternativas para el reconocimiento de las pérdidas esperadas, el IASB ha desarrollado un modelo que aboga por 
la implantación de unas políticas de reconocimiento caracterizadas por un mayor conservadurismo incondicional. El cambio regulatorio propuesto introduce una visión del concepto de activo influenciado por este conservadurismo incondicional ya que recoge desde el mismo comienzo las estimaciones futuras de las pérdidas que probablemente experimente el activo crediticio, y que tiene como resultado una información financiera más prudente y con menor volatilidad en los resultados.

En nuestra opinión, este proceso supone la introducción de mayores niveles de prudencia que solo encontrarían justificación en el marco conceptual si se considerara que la probabilidad de ocurrencia de esas pérdidas es alta con base en el comportamiento histórico de los activos financieros. Sin embargo, la elevada incertidumbre que entrañan el momento y la cantidad que representarán tales pérdidas, puede implicar una merma en el objetivo de representación fiel de la información financiera, al menos en algunos períodos.

Esta reducción en el objetivo de representación fiel se ha justificado en la necesidad de protección de acreedores e inversores, en especial en el sistema financiero, cuya estabilidad se ha visto amenazada con la reciente crisis financiera. No obstante, coincidimos con las opiniones que ven en este proceso un intento de la información financiera por arrogarse la función de supervisión del sistema bancario. En este sentido, las normas de solvencia bancaria incluyen un grado de análisis de la cartera crediticia, de sus riesgos y garantías, muy exhaustivo al que no han llegado aún los sistemas de cálculo propuestos por el IASB, limitados por el argumento coste-beneficio.

Por tanto, el desacoplamiento entre la información financiera y las normas de solvencia en las entidades de crédito permanece en la situación actual de la reforma de la NIIF 9. Ello obligará al supervisor — que sí tiene inequívocamente asignada la función de garantizar la continuidad del sistema financiero- - a adaptar sus disposiciones para la definición de la solvencia mínima, a partir de las nuevas normas de información financiera.

\section{Referencias}

Ahmed, Anwer S.; Takeda, Carolyn \& Thomas, Shawn (1999). Bank Loan Loss Provision.

A Reexamination of Capital Management, Earnings Management and Signaling Effects. Journal of Accounting and Economics, 28 (1), 1-26.

Akerlof, George A. (1970). The Market for 'Lemons': Quality Uncertainty and the Market Mechanism. The Quarterly Journal of Economics, 84 (3), 488-500.

Albertazzi, Ugo \& Gambacorta, Leonardo (2009). Bank Profitability and the Business Cycle. Journal of Financial Stability, 5 (4), 393-409.

Allen, Franklin \& Carletti, Elena (2008). Mark-to-Market Accounting and Liquidity Pricing. Journal of Accounting and Economics, 45 (2), 358-378. Disponible en: http://apps. eui.eu/Personal/Carletti/Allen-CarlettiMMA-final.pdf Anagnostopoulos, Yiannis \& Buckland, Roger (2005). Historical Cost versus Fair Value 
Accounting in Banking: Implications for Supervision, Provisioning, Financial Reporting and Market Discipline. Journal of Banking Regulation, 6 (2), 109-127.

Anandarajan, Asokan; Hasan, Iftekhar \& Lozano-Vivas, Ana (2003). The Role of Loan Loss Provisions in Earnings Management, Capital Management and Signaling: The Spanish Experience. Advances in International Accounting, 16, 45-65.

Arcas-Pellicer, María José (1994). Reacción del precio de las acciones a la publicación de los beneficios anuales: análisis empírico en el sector bancario. Revista Española de Financiación y Contabilidad, 24 (78), 181201. Disponible en: http://www.aeca.es/ pub/refc/articulos.php? id=0439

Banco de España (2004). Circular 4/2004, entidades de crédito: normas de información financiera pública y reservada, y modelos de estados financieros. Disponible en: http:// www.bde.es/f/webbde/SJU/normativa/ circulares/4.2004.pdf

Bank for International Settlements, BIS (1988). Basilea I: Convergencia internacional de medidas y normas de capital. Basilea, Suiza: Bank for International Settlements, BIS. Marco revisado, versión integral de 2006, disponible en: http://www.bis.org/ publ/bcbs128_es.pdf

Bank for International Settlements, BIS (2001). Basilea II: El Nuevo Acuerdo de Capital de Basilea. Basilea, Suiza: Bank for International Settlements, BIS. Disponible en: http://www.bis.org/publ/bcbsca03_s.pdf Bank for International Settlements, BIS (2010). Basilea III: marco regulador global para reforzar los bancos y sistemas bancarios. Basilea, Suiza: Bank for International Settlements, BIS. Disponible en: http:// www.bis.org/publ/bcbs189_es.pdf Basu, Sudipta (1997). The Conservatism Principle and the Asymmetric Timeliness of Earnings. Journal of Accounting and Economics, 24 (1), 3-37.

Beatty, Anne; Chamberlain, Sandra L. \& Magliolo, Joseph (1995). Managing Financial Reports of Commercial Banks: the Influence of Taxes, Regulatory Capital and Earnings. Journal of Accounting Research, 33 (2), 231-261.

Beaver, William H. \& Engel, Ellen E. (1996). Discretionary Behavior with Respect to Allowances for Loan Losses and the Behavior of Security Prices. Journal of Accounting and Economics, 22 (1), 177-206. Beekes, Wendy; Pope, Peter \& Young, Steven (2004). The Link between Earnings Timeliness, Earnings Conservatism and Board Composition: Evidence from the UK. Corporate Governance: An International Review, 12 (1), 47-59.

Biddle, Gary C.; Ma, Mary L. Z. \& Wu, Feng (2012). Conditional Conservatism and the Cost of Equity Capital: Informational, Fundamental, and Behavioral Effects. Fundamental and Behavioral Effects (January 15, 2012). Disponible en: http://asianfa2012. mcu.edu.tw/fullpaper/10311.pdf Bikker, Jacob A. \& Metzemakers, Paul A. J. (2005). Bank Provisioning Behaviour and Procyclicality. Journal of International Financial Markets, Institutions and Money, 15 (2), 141-157. 
Blose, Laurence E. (2001). Information Asymmetry, Capital Adequacy and Market Reaction to Loan Loss Provision Announcements in the Banking Industry. The Quarterly Review of Economics and Finance, 41 (2), 239-258.

Borio, Claudio \& Tsatsaronis, Kostas (2004). Accounting and Prudential Regulation: from Uncomfortable Bedfellows to Perfect Partners? Journal of Financial Stability, 1 (1), 111-135.

Borio, Claudio \& Tsatsaronis, Kostas (2005). Accounting, Prudential Regulation and Financial Stability: Elements of a Synthesis (180). BIS Working Paper, Bank for International Settlements. Disponible en http://www.bis.org/publ/work180.htm Boyer, Robert (2007). Assessing the Impact of Fair Value upon Financial Crisis. Socio Economy Review, 5 (4), 779-807.

Cano-Rodríguez, Manuel (2010). Big Auditors, Private Firms and Accounting Conservatism: Spanish Evidence. European Accounting Review, 19 (1), 131-159.

Cornett, Marcia Millon; McNutt, Jamie John \& Tehranian, Hassan (2009). Corporate Governance and Earnings Management at large U.S. Bank Holding Companies. Journal of Corporate Finance, 15 (4), 412-430. Disponible en: https:// www2.bc.edu/ tehranih/Corporate_ Governance.pdf

Cortavarria, Luis; Dziobek, Claudia; Kanaya, Akihiro \& Song, Inwon (2000). Loan Review, Provisioning, and Macroeconomic Linkages (WP/00/195). IMF Working
Paper. Disponible en: https://www.imf.org/ external/pubs/ft/wp/2000/wp00195.pdf

Dechow, Patricia M. \& Skinner, Douglas J. (2000). Earnings Management: Reconciling the Views of Accounting Academics, Practitioners, and Regulators. Accounting Horizons, 14 (2), 235-250.

Dechow, Patricia M.; Sloan, Richard G. \& Sweeney, Amy P. (1996). Causes and Consequences of Earnings Manipulation: An Analysis of Firms Subject to Enforcement Actions by the SEC. Contemporary Accounting Research, 13 (1), 1-36.

Deschênes, Sebastien (2008). Loan Loss Estimation Management by Financial Institution Managers and Commercial Loan Officers. Journal of Performance Management, 3, 16-31.

Dugan, John C. (2009). Loan Loss Provisioning and Pro-cyclicality. Remarks by John C. Dugan, Comptroller of the Currency, before the Institute of International Bankers. Disponible en: http://www.occ.gov/news-issuances/ speeches/2009/pub-speech-2009-16.pdf Financial Accounting Standards Board, FASB (2010). Proposed Accounting Standards Update, PASU. Accounting for Financial Instruments and Revisions to the Accounting for Derivative Instruments and Hedging Activities. Norwalk. Mayo. Disponible en: http://www.fasb.org/cs/ BlobServer?blobkey $=$ id\&blobnocache $=$ true \&blobwhere $=1175823559151$ \&blobheader $=\mathrm{a}$ pplication\%2Fpdf\&blobheadername2=Cont ent-Length\&blobheadername $1=$ Content-Dis position\&blobheadervalue $2=842688 \&$ blobh eadervalue1=filename\%3DProposed_ASU_ Derivatives_and_Hedging_Topic_815 
and_Financial_Instruments_Topic_825_.pd f\&blobcol $=$ urldata\&blobtable $=$ MungoBlobs

Financial Accounting Standards Board, FASB (2008). Statement of Financial Accounting Concepts, SFAC 2. Qualitative Characteristics of Accounting Information. Disponible en: http://www.fasb.org/cs/BlobServer?blobcol $=$ urldata\&blobtable $=$ MungoBlobs\&blobkey $=$ id\&blobwhere $=1175820900526 \&$ blobhea der=application $\% 2 \mathrm{Fpdf}$

Financial Stability Forum, FSB (2008). Report of the Financial Stability Forum on Enhancing Market and Institutional Resilience. Basilea. Abril. Disponible en: http:/www.financialstabilityboard.org/ publications/r_0804.pdf.

Fonseca, Ana Rosa \& González, Francisco (2008). Cross-country Determinants of Bank Income Smoothing by Managing Loan-Loss Provisions. Journal of Banking \& Finance, 32 (2), 217-228.

Francis, Bill; Hasan, Iftekhar \& Wu, Qiang (2013). The Benefits of Accounting Conservatism to Shareholders: Evidence from the Financial Crisis. Accounting Horizons, 27 (2), 319-346. Disponible en: http://dx.doi.org/10.2139/ssrn.2291352.

García-Lara, Juan Manuel; García-Osma, Beatriz \& Peñalva, Fernando (2009). Accounting Conservatism and Corporate Governance. Review Accounting Studies, 14, 161-201.

García-Lara, Juan Manuel; García-Osma, Beatriz \& Peñalva, Fernando (2011). Conditional Conservatism and Cost of Capital. Review of Accounting Studies, 16 (2), 1-41. Disponible en: http://papers.ssrn.com/ sol3/papers.cfm?abstract_id=1544307
Gebhardt, Günther; Reichardt, Rolf \& Wittenbrink, Carsten (2004). Accounting for Financial Instruments in the Banking Industry: Conclusions from a Simulation Model. European Accounting Review, 13 (2), 341-371. Disponible en: https://www.econstor.eu/dspace/ bitstream/10419/72631/1/03_21_revised.pdf Goldin, Ian \& Vogel, Tiffany (2010). Global Governance and Systemic Risk in the $21^{\text {st }}$ Century: Lessons from the Financial Crisis. Global Policy, 1 (1), 4-15. Disponible en: http://onlinelibrary.wiley.com/doi/10.1111/ j.1758-5899.2009.00011.x/pdf González-Mota, Emiliano (2005).

Prociclicidad, volatilidad financiera y Basilea II. Revista de Estabilidad Financiera, 8, 151-163. Disponible en: http://www.bde.es/f/webbde/Secciones/ Publicaciones/InformesBoletinesRevistas/ RevistaEstabilidadFinanciera/05/Fich/ estfin08_rev.pdf

Hartwig, Fredrik (2013). Preparers and Nonpreparers Lobbying on the Proposed Prohibition of Goodwill Amortisation in ED3 Business Combinations. The Finnish Journal of Business Economics, 63 (3-4), 30 60. Disponible en: http://du.diva-portal.org/ smash/get/diva2:623074/FULLTEXT01.pdf Hellwig, Martin F. (2009). Systemic Risk in the Financial Sector: An Analysis of the Subprime-Mortgage Financial Crisis. De Economist, 157 (2), 129-207. Disponible en: http://www.parisschoolofeconomics.eu/ docs/guesnerie-roger/hellwig.pdf 
Herz, Robert H. (2010). Accounting Standards and Bank Regulation. International Journal of Disclosure and Governance, 7 (2), 97-107. International Accounting Standards Board, IASB (2003). Instrumentos financieros: Reconocimiento y valoración. Londres. Disponible en: http://www. normasinternacionalesdecontabilidad.es/ nic/pdf/NIC39.pdf

International Accounting Standards Board, IASB (2008). Discussion Paper. Reducing Complexity in Reporting Financial Instruments. Londres. Marzo. Disponible en: http://www.ifrs.org/ Current-Projects/IASB-Projects/FinancialInstruments-A-Replacement-of-IAS39-Financial-Instruments-Recognitio/ Discussion-Paper-and-Comment-Letters/ Documents/DPReducingComplexity_ ReportingFinancialInstruments.pdf International Accounting Standards Board, IASB (2009a). Request for information. ('Expected Loss Model') Impairment of Financial Assets: Expected Cash Flow Approach. Junio. Londres.

Disponible en: http://www.ifrs.org/ Current-Projects/IASB-Projects/ Financial-Instruments-A-Replacement-ofIAS-39-Financial-Instruments-Recognitio/ Impairment/InfoRequest/Documents/ RequestforInformation.pdf International Accounting Standards Board, IASB (2009b). Exposure Draft: Financial Instruments: Amortised Cost and Impairment. Londres. Noviembre. Disponible en: http://www.ifrs.org/Current-Projects/ IASB-Projects/Financial-Instruments-
A-Replacement-of-IAS-39-FinancialInstruments-Recognitio/Impairment/ED/ Documents/vbEDFIImpairmentNov09.pdf International Accounting Standards Board, IASB (2010). The Conceptual Framework for Financial Reporting. Septiembre. Disponible en: http://www. ifrs.org/News/Press-Releases/Documents/ ConceptualFW2010vb.pdf International Accounting Standards Board, IASB (2013a). Exposure Draft. Financial Instruments: Expected Credit Losses. Londres. Marzo. Disponible en: http://www.ifrs. org/Current-Projects/IASB-Projects/ Financial-Instruments-A-Replacement-ofIAS-39-Financial-Instruments-Recognitio/ Impairment/Exposure-Draft-March-2013/ Comment-letters/Documents/EDFinancial-Instruments-Expected-CreditLosses-March-2013.pdf International Accounting Standards Board, IASB (2013b). Staff Paper. Financial Instruments: Impairment. Disponible en http://www.ifrs.org/Meetings/ MeetingDocs/IASB/2013/July/05CImpairment.pdf

International Accounting Standards Board, IASB \& Financial Accounting Standards Board, FASB (2011). Supplement to Exposure Draft. Joint Proposed Approach to Accounting for Credit Losses. Londres. Enero. Disponible en: http://www.ifrs. org/Current-Projects/IASB-Projects/ Financial-Instruments-A-Replacement-ofIAS-39-Financial-Instruments-Recognitio/ Impairment/Supplementdoc/Documents / 
SupplementarydocFinancialInstruments ImpairmentJan2011.pdf

Jiménez, Gabriel \& Saurina, Jesús (2005). Credit Cycles, Credit Risk, and Prudential Regulation. Documentos de trabajo del Banco de España, 31, 8-34. Disponible en: http:// www.bde.es/f/webbde/SES/Secciones/ Publicaciones/PublicacionesSeriadas/ DocumentosTrabajo/05/Fic/dt0531e.pdf Kanagaretnam, Kiridaran; Lobo, Gerald J. \& Yang, Dong Hoon (2005). Determinants of Signaling by Banks through Loan Loss Provisions. Journal of Business Research, 58 (3), 312-320.

Kim, Myungsun \& Kross, William (1998). The Impact of the 1989 Change in bank Capital Standards on Loan Loss Provisions and Loan Write-offs. Journal of Accounting and Economics, 25, 69-99.

Kothari, S. P. \& Lester, Rebecca (2012). The Role of Accounting in the Financial Crisis: Lessons for the Future. Accounting Horizons, 26 (2), 335-351.

Laeven, Luc \& Majnoni, Giovanni (2003). Loan Loss Provisioning and Economic Slowdowns: Too Much, Too Late? Journal of Financial Intermediation, 12 (2), 178-197. Liu, Chi-Chun \& Ryan, Stephen G. (2006). Income Smoothing over the Business Cycle: Changes in Banks' Coordinated Management of Provisions for Loan Losses and Loan Charge-offs from the Pre-1990 Bust to the 1990s Boom. The Accounting Review, 81 (2), 421-441.

Lobo, Gerald J. \& Yang, Dong Hoon (2001). Bank Managers' Heterogeneous Decisions on Discretionary Loan Loss Provisions.
Review of Quantitative Finance and Accounting, 16 (3), 223-250.

Matherat, Sylvie (2008). Fair Value Accounting and Financial Stability: Challenges and Dynamics. Financial Stability Review, 12, 53-64. Disponible en: http://www.banque-france.fr/ fileadmin/user_upload/banque_de_france/ publications/Revue_de_la_stabilite_ financiere/etud6_1008.pdf

Mohammed, Nor Farizal; Ahmed, Kamran \& Ji, Xu Dong (2010). Accounting Conservatism, Corporate Governance and Political Influence: Evidence from Malaysia. Working paper, Disponible en: http://baa. group.shef.ac.uk/download_papers/index. php?submit $=$ download_file\&username $=$ ba fa\&token $=26684 \mathrm{~b} 3867876 \mathrm{df7bd} 3 \mathrm{fcac} 34 \mathrm{~d} 8$ cbddb\&paper_id=134

Pérez, Daniel; Salas-Fumás, Vicente \& Saurina, Jesús (2008). Earnings and Capital Management in Alternative Loan Loss Provision Regulatory Regimes. European Accounting Review, 17 (3), 423-445.

Persaud, Avinash D. (2008). Regulation, Valuation, and Systemic Liquidity. Financial Stability Review, 12, 75-83. https://www. banque-france.fr/fileadmin/user_upload/ banque_de_france/publications/Revue_de_ la_stabilite_financiere/etud8_1008.pdf Petersen, Mitchell A. \& Thiagarajan, S. Ramu (2000). Risk Measurement and Hedging: With and Without Derivatives. Financial Management, 29 (4), 5-29.

Plantin, Guillaume; Sapra, Haresh \& Shin, Hyun Song (2008). Fair Value Accounting and Financial Stability. Financial Stability 
LA CONTABILIDAD ANTE LA CRISIS FINANCIERA / I. DE CABO, R. BAUTISTA, H. MOLINA, J. RAMíREZ / 395

Review, 12, 84-94. Disponible en: https:// www.princeton.edu/ hsshin/www/

BdFFSRmtm.pdf

Qiang, Xinrong (2007). The Effects of Contracting, Litigation, Regulation, and Tax Costs on Conditional and Unconditional Conservatism: CrossSectional Evidence at the Firm Level. The Accounting Review, 82 (3), 759-796.

Rueda, Juan Antonio (2006). Prudencia del resultado bajo normas internacionales de contabilidad. Un estudio comparado en la UE. Cuadernos de Estudios Empresariales, 16, 55-79. Disponible en: http://revistas. ucm.es/index.php/CESE/article/ viewFile/CESE0606110055A/9473, http://dialnet.unirioja.es/servlet/ ejemplar?codigo $=153207$

Shen, Chung Hua \& Chih, Hsiang Lin (2005). Investor Protection, Prospect Theory, and Earnings Management: An International Comparison of the Banking Industry. Journal of Banking \& Finance, 29 (10), 2675-2697.

Song, Guoxiang (2011). The Benefits of Decoupling Financial Reporting from Bank Capital Regulation. Disponible en: http:// dx.doi.org/10.2139/ssrn.1955453

Song, Guoxiang (2012). Can Accounting Rules Be Made Neutral for Bank Capital Regulation? Journal of Governance and Regulation, 1 (3), 27-35. Disponible en: http://papers.ssrn.com/sol3/papers. cfm?abstract_id=1999467
Wahlen, James M. (1994). The Nature of Information in Commercial Bank Loan Loss Disclosures. The Accounting Review, 69 (3), 455-478.

Watts, Ross L. (2003a). Conservatism in Accounting - Part I: Explanations and Implications. Accounting Horizons, 17 (3), 207-221. http://papers.ssrn.com/sol3/ papers.cfm?abstract_id $=414522$

Watts Ross L. (2003b). Conservatism in Accounting - Part II: Evidence and Research Opportunities. Accounting Horizons, 17 (3), 287-301. Disponible en: http://papers.ssrn.com/sol3/papers. cfm?abstract_id $=438662$

Watts, Ross L. \& Zimmerman, Jerold L. (1983). Agency Problems, Auditing, and the Theory of the Firm: Some Evidence. Journal of Law and Economics, 26 (3), 613-633.

- Fecha de recepción: 30 de octubre de 2013

- Fecha de aceptación: 29 de abril de 2014

- Disponible en línea: 01 de julio de 2014

\section{Para citar este artículo}

Cabo-Moreno, Ignacio de; Molina-Sánchez, Horacio; Ramírez-Sobrino, Jesús N. \& Bautista-Mesa, Rafael (2014). El papel de la contabilidad ante la crisis financiera. Una reflexión sobre el conservadurismo incondicional de la NIIF 9. Cuadernos de Contabilidad, 15 (38), 371-395.

doi: 10.11144/Javeriana.cc15-38.pccf 
